\title{
Specify Other Inhalation Anesthesia Agents
}

National Cancer Institute

\section{Source}

National Cancer Institute. Specify Other Inhalation Anesthesia Agents. NCI Thesaurus. Code C159584.

A request to specify inhalation anesthesia that was administered but not included on a list. 